\title{
Transmission power control game with SINR as objective function *
}

\author{
E. Altman ${ }^{1}, \mathrm{~K}$. Avrachenkov ${ }^{1}$, and A. Garnaev ${ }^{2}$ \\ 1 INRIA Sophia Antipolis, France \\ \{altman, k.avrachenkov\}@sophia.inria.fr \\ 2 St. Petersburg State University, Russia \\ agarnaev@rambler.ru
}

\begin{abstract}
We consider the transmission power control problem with SINR as objective function in the two scenarii: selfish and cooperative. We show that in the selfish (non-cooperative) scenario several Nash equilibria can arise. In particular, the game can take the form of the HawkDove game, where the users can choose either conciliation or conflict fighting for shared sub-carriers. We fully characterize different types of Nash equilibria. In the cooperative scenario, we show that the parameter area where users employ pure strategies is essentially narrower than the area where users employ mixed strategies. Moreover, we identify an area where Nash equilibrium and Pareto equilibrium coincide. If one of the users has a big signal to transmit (called a stronger user) for both scenarious meanwhile his rival has small signal to transmit (weaker user) then behaviour of the stronger user drastically changes in cooperative plot compare to selfish one. Namely, in selfish one the stronger user squeezes the weaker one from the best channel meanwhile in cooperative he allows the weaker user employs the best channel and himself prefers to apply a mixed strategy.
\end{abstract}

Keywords: Wireless networks, Power Control, Nash Equilibrium, Cooperation

\section{Introduction}

We consider the transmission power control problem with SINR as objective function in the three scenarii: selfish and cooperative. In particular, in the selfish scenario we consider two users who try to send information through $n$ resources. The strategy of user $j(j=1,2)$ is $T^{j}=\left(T_{1}^{j}, \ldots, T_{n}^{j}\right)$ with $T_{i}^{j} \geq 0$ such that $\sum_{i=1}^{n} \pi_{i} T_{i}^{j}=\bar{T}^{j}$, where $\bar{T}^{j}>0$. Here we assume that resource $i$ has a "weight" of $\pi_{i}>0$. The resources may correspond to capacity available at different time slots; we assume that there is a varying environment whose state changes among a finite set of states $i \in[1, n]$, according to some ergodic stochastic process with

\footnotetext{
* The work was supported by EGIDE ECO-NET grant no.18933SL "Game Theory
} for Wireless Networks" and RFBR and NNSF Grant no.06-01-39005. 
stationary distribution $\left\{\pi_{i}\right\}$. Either the resources may correspond to frequency bands (e.g. as in OFDM) where one should assign different power levels for different sub-carriers [9]. In that case we may take $\pi_{i}=1 / n$ for all $i$.

In the selfish (non-cooperative) scenario each user tries to maximize its average SINR:

$$
v^{j}\left(T^{1}, T^{2}\right)=\sum_{i=1}^{n} \pi_{i} \frac{\alpha_{i}^{j} T_{i}^{j}}{N_{i}^{0}+\alpha_{i}^{\hat{j}} T_{i}^{\hat{j}}},
$$

where $N_{i}^{0}$ is the noise level and $\alpha_{i}^{j}>0$ are fading channel gains of user $j$ when the environment is in state $i$, and $\hat{j}=3-j$. We assume that all the fading channel gains $\alpha_{i}^{j}$, the noise levels $N_{i}^{0}$, the total powers $\bar{T}^{j}$ are known to both users or they can be quickly inferred [4]. The authors of [1] have studied the case of incomplete information.

The SINR as an objective function in the power control game was also considered in [6]: all users have a single common channel and choose between several base stations. We note that in the regime of low SINR the present objective can serve as an approximation to the Shannon capacity. A central motivation to consider SINR as an objective function and not Shannon capacity, is that current technology for voice over wireless does not try to achieve Shannon capacity but rather uses given codecs that can adapt the transmission rate to the SINR; these turn out to adapt the rate in a way that is linear in the SINR over a wide range of throughput. The SINR has therefore been used very often to represent directly the throughput see $[7,8]$. The validity of this can be seen e.g. in [5, p. 151, 222, 239]. As we see from [5, Fig. 10.4, p. 222], the ratio between the throughput and the SINR is close to a constant throughout long range of bit rates. For example, between $16 \mathrm{Kbps}$ and $256 \mathrm{Kbps}$, the maximum variation around the median value is less than $20 \%$.

We finally note that with an SINR objective we are able to characterize fully cooperative and non-cooperative. In [2] with the Shannon capacity as an objective we have dealt only with the symmetric case. An interested reader can find more relevant literature on Gaussian Interference Game in $[2,4]$. In the present work we restrict ourselves to the case of two users. We believe that the two user case is good for illustration and many techniques of this paper apply to the case of more than two users which we leave as a topic for future research.

In the non-cooperative scenario we look for a NE (Nash Equilibrium), that is, we want to find such couple of strategies $\left(T^{1 *}, T^{2 *}\right)$ that for any $\left(T^{1}, T^{2}\right)$ the inequalities hold: $v^{1}\left(T^{1}, T^{2 *}\right) \leq v^{1}\left(T^{1 *}, T^{2 *}\right), \quad v^{2}\left(T^{1 *}, T^{2}\right) \leq v^{2}\left(T^{1 *}, T^{2 *}\right)$.

In the cooperative scenario, both users want to maximize $v_{1}+v_{2}$. We shall show that in some parameter settings cooperative and non-cooperative strategies coincide.

It is worth to note that in [3] one of results concerns a game which can be considered as a game with the SINR as object function in the jamming scenario the first user wants to maximize the objective function $v_{1}$ and the second user wants to minimize this objective function. For this game the jammer in his optimal behaviour tends to equalize the quality of the best sub-carriers to as low level as his power constraint allows. 
We will assume that all channels differ by their quality for both users. Namely, we assume that for each user $j(j=1,2) \alpha_{i_{1}}^{j} / N_{i_{1}}^{0} \neq \alpha_{i_{2}}^{j} / N_{i_{2}}^{0}$ for any $i_{1} \neq i_{2}$. Without lost of generality we can assume that $\{a(j, 1), a(j, 2), \ldots, a(j, n)\}$ is a permutation of $\{1,2, \ldots, n\}$ such that the sub-carriers are arranged in the following decreasing order by their quality: $\alpha_{a(j, 1)}^{j} / N_{a(j, 1)}^{0}>\ldots>\alpha_{a(j, n)}^{j} / N_{a(j, n)}^{0}$.

\section{Non-cooperative scenario}

\subsection{The best sub-carriers differs for the users}

Since $v^{i}$ is linear in $T^{i}$ the optimal strategy of user $i$ can be nonnegative only for sub-carriers where the compound SINR is maximal. So, we have the following result describing the structure of the optimal strategies.

Theorem $1\left(T^{1}, T^{2}\right)$ is a Nash equilibrium if and only if there are non-negative $\omega^{1}$ and $\omega^{2}$ (which present the maximal compound SINR for the corresponding user) such that for $j=1,2$ and $i \in[1, n]$ :

$$
T_{i}^{j} \geq 0 \text { for } \frac{\alpha_{i}^{j}}{N_{i}^{0}+\alpha_{i}^{\hat{j}} T_{i}^{\hat{j}}}=\omega^{j} \text { and } T_{i}^{j}=0 \text { for } \frac{\alpha_{i}^{j}}{N_{i}^{0}+\alpha_{i}^{\hat{j}} T_{i}^{\hat{j}}}<\omega^{j} .
$$

The payoffs corresponding to these strategies are $\left(\omega^{1} \bar{T}^{1}, \omega^{2} \bar{T}^{2}\right)$.

The strategy when user $j(j=1,2)$ transmits all the signal through just one sub-carrier (say, $i$ ) will be called a pure one and we will denote it by $\mathcal{T}_{i}^{j}$. So, the pure strategy is $\mathcal{T}_{i}^{j}=\left(T_{1}^{j}, \ldots, T_{n}^{j}\right)$ such that $T_{k}^{j}=\bar{T}^{j}$ for $k=i$ and $T_{k}^{j}=0$ otherwise. The strategies when users employ more than one sub-carrier to transmit signal will be called mixed strategies.

If the best sub-carriers are different for users, namely $a(1,1) \neq a(2,1)$, then by Theorem 1 the NE has time sharing form, namely the following result holds.

Theorem 2 If $a(1,1) \neq a(2,1)$, then the game has the unique $N E$ and it is the pure one $\left(\mathcal{T}_{a(1,1)}^{1}, \mathcal{T}_{a(2,1)}^{2}\right)$.

It is worth to note that if there are more than one best quality sub-carrier the NE is not unique at all since there is enough room for the users to share these best quality channels.

So, our assumption that all sub-carriers are different by its quality is quite reasonable. But as it will be shown in the next section even then if the the best sub-carriers coincides a variety of $\mathrm{NE}$ is possible.

\subsection{The best first sub-carriers coincides for users}

Let the first best sub-carriers coincides for users, i.e. $a(1,1)=a(2,1)=a(1)$. Then some variety of cases arise depending on the quality of the second best sub-carriers and the power of signal the users can apply. 
The SINRs for the best sub-carrier with the induced noise are big enough for both users:

If the SINRs for the best sub-carrier with the induced noise are greater than the SINRs with the natural noise for the second best sub-carriers for both users, then each of them could manage to transmit all the signal just through the best sub-carrier. The next theorem follows straighforward from Theorem 1 and supplies the formulas describing the corresponding conditions on SINRs.

Theorem 3 Let the following inequalities hold

$$
\frac{\alpha_{a(1)}^{j}}{N_{a(1)}^{0}+\alpha_{a(1)}^{\hat{j}} \bar{T}^{\hat{j}}} \geq \frac{\alpha_{a(j, 2)}^{j}}{N_{a(j, 2)}^{0}} \text { for } j=1,2 .
$$

Then there is the unique $N E$ and it is the pure one $\left(\mathcal{T}_{a(1)}^{1}, \mathcal{T}_{a(1)}^{2}\right)$.

Note that inequalities (1) can be rewritten in the following equivalent form

$$
A^{j} \geq \bar{X}^{j} \text { for } j=1,2,
$$

where $A^{\hat{j}}=\alpha_{a(1)}^{j} N_{a(j, 2)}^{0} / \alpha_{a(j, 2)}^{j}-N_{a(1)}^{0}$ and $\bar{X}^{j}=\alpha_{a(1)}^{j} \bar{T}^{j}$.

The SINR for the best sub-carrier with the induced noise is big enough only for one user:

If the SINR for the best sub-carrier with the induced noise is greater than the SINR with the natural noise for the second best sub-carriers only for one user (say, user $\hat{j}$ ), then the other user (user $j$ ) could threaten to jam the best sub-carrier and user $\hat{j}$ has nothing to threaten back. This makes him to withdraw and to use the second best sub-carrier. The next theorem follows straighforward from Theorem 1 and supplies the formulas describing the corresponding relations between SINRs.

Theorem 4 Let $A^{j}>\bar{X}^{j}, \quad A^{\hat{j}}<\bar{X}^{\hat{j}}$ hold either for $j=1$ or $j=2$ : Then the game has the unique $N E$ and it is the pure one $\left(\mathcal{T}^{1}, \mathcal{T}^{2}\right)$ where $\mathcal{T}^{j}=\mathcal{T}_{a(j, 2)}^{j}$ and $\mathcal{T}^{\hat{j}}=\mathcal{T}_{a(1)}^{\hat{j}}$.

Thus, the weaker user is squeezed out from the common best sub-carrier by the threat from the stronger user.

The SINRs for the best sub-carrier with the induced noise are small for both users:

If the SINRs for the best sub-carrier with the induced noise are less than the SINRs with the natural noise for the second best sub-carriers for the both users, then each user could threaten to jam the best sub-carrier for his opponent. The 
situation becomes very competitive and Hawk-Dove type strategies are possible. The next theorem follows straighforward from Theorem 1 and supplies the formulas describing the corresponding relations between SINRs and Hawk-Dove type strategies.

Theorem 5 Let $A^{j}<\bar{X}^{j}$ hold for $j=1,2$. Then there are two pure $N E$ equilibria $\left(\mathcal{T}_{a(1)}^{1}, \mathcal{T}_{a(2,2)}^{2}\right)$ and $\left(\mathcal{T}_{a(1,2)}^{1}, \mathcal{T}_{a(1)}^{2}\right)$.

In the first pure NE user 1 presents an aggressive (Hawk) player always fighting for the best quality sub-carrier meanwhile user 2 is a withdrawing (Dove) player escaping any fighting. In the second pure NE the users exchange the roles. On Figure 1 possible pure NE are pointed out depending on power signals they have to transmit.

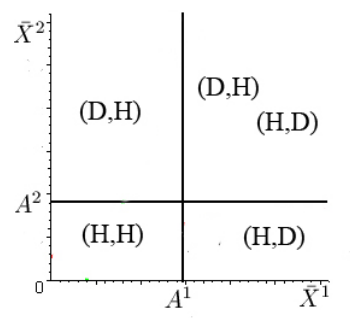

Fig. 1. Pure NE strategies

Besides the two pure NE a mixed NE can take place. Let us consider the case when the second best sub-carriers are different for users. Then by Theorem 1 we can see that in the mixed equilibrium both users at first take care about the opponent by equalizing quality of the opponent's two best by quality subcarriers. It allows users to transmit all the rest of the signal through the second best sub-carriers. Of course, this strategy assumes some cooperation between the users.

Theorem 6 Let $A^{j}<\bar{X}^{j}$ hold for $j=1,2$ hold and $a(1,2) \neq a(2,2)$, so the second best sub-carriers are different for users. Then there is the unique mixed $N E$, using only two first by quality sub-carriers, given by the following strategies which equalize the first and second by quality sub-carriers and transmit the rest power through the second sub-carriers:

$$
T_{i}^{j}= \begin{cases}\frac{\alpha_{a(1)}^{\hat{j}}}{\alpha_{a(1)}^{j}}\left(1 / \omega^{\hat{j}}-N_{a(1)}^{0} / \alpha_{a(1)}^{\hat{j}}\right), & \text { for } i=a(1), \\ \bar{T}^{j}-T_{a(1)}^{j}, & \text { for } i=a(j, 2), \\ 0, & \text { otherwise, }\end{cases}
$$

where $\omega^{j}=\alpha_{a(j, 2)}^{j} / N_{a(j, 2)}^{0}, j=1,2$. 


\subsection{The $K$ best sub-carriers coincide:}

In this subsection we consider situation when the $K(K \leq n)$ best subcarriers coincide for users, i.e. $a(1, i)=a(2, i)=a(i), i \in[1, K]$ and $a(1, K+1) \neq$ $a(2, K+1)$ if $K<n$. Theorem 6 can be generalized as follows:

Theorem 7 Let $k^{1}=k^{2}<K$ where $k^{j}(j=1,2)$ are integers such that

$$
H^{\hat{j}}\left(\alpha_{a\left(j, k^{j}\right)}^{j} / N_{k^{j}}^{0}\right)<\bar{T}^{\hat{j}} \leq H^{\hat{j}}\left(\alpha_{a\left(j, k^{j}+1\right)}^{j} / N_{k^{j}+1}^{0}\right),
$$

where $\alpha_{a(j, n+1)}^{j} / N_{n+1}^{0}=0$ and

$$
H^{\hat{j}}(\omega)=\sum_{i=1}^{n} \frac{\alpha_{a(j, i)}^{j}}{\alpha_{a(j, i)}^{\hat{j}}}\left[1 / \omega^{j}-N_{a(j, i)}^{0} / \alpha_{a(j, i)}^{j}\right]_{+} .
$$

Then the NE is given by the strategies $\left(T^{1}, T^{2}\right)$ where

$$
T_{i}^{\hat{j}}=\frac{\alpha_{a(i)}^{j}}{\alpha_{a(i)}^{\hat{j}}}\left[1 / \omega^{j}-N_{a(i)}^{0} / \alpha_{a(i)}^{j}\right]_{+}, \quad i \in[1, n]
$$

for $j=1,2$, and $\omega^{j}$ is the unique root of the equation $H^{\hat{j}}\left(\omega^{j}\right)=\bar{T}^{\hat{j}}$.

Thus, if the powers of signal to transmit are approximately equal then the equilibrium mixed strategies have water-filling structure.

Theorem 8 Let $K=k^{1}=k^{2}$ and $a(1, K+1) \neq a(1, K+1)$. Then if the power of signals allows to equalize the first $K+1$ channels, so

$$
H^{\hat{j}}\left(\alpha_{a(j, K+1)}^{j} / N_{a(j, K+1)}^{0}\right)<\bar{T}^{\hat{j}}
$$

then the NE is given by the strategies $\left(T^{1}, T^{2}\right)$ where

$$
T_{i}^{j}= \begin{cases}\frac{\alpha_{a(i)}^{\hat{j}}}{\alpha_{a(i)}^{j}}\left[1 / \omega^{\hat{j}}-N_{a(i)}^{0} / \alpha_{a(i)}^{\hat{j}}\right]_{+} & \text {for } i=a(1), \ldots, a(k), \\ \bar{T}^{j}-H^{j}\left(\omega^{\hat{j}}\right) & \text { for } i=a(j, K+1), \\ 0 & \text { otherwise, }\end{cases}
$$

where $\omega^{j}=\alpha_{a(j, K+1)}^{j} / N_{a(j, K+1)}^{0}, j=1,2$. 


\section{A cooperative scenario}

In this section we assume that the users cooperate and they want to maximize their joint payoff $v=v^{1}+v^{2}$. Of course if the best sub-carriers are different for users, then, time sharing strategies $\mathcal{T}_{a(1,1)}^{1}, \mathcal{T}_{a(2,1)}^{2}$ are the optimal one. Next let us consider the case when the best sub-carriers coincide for the users, namely $a(1,1)=a(2,1)=a(1)$. The next theorem supplies the complete solution of this problem where the second best sub-carriers are different, namely $a(1,2) \neq$ $a(2,2)$.

Theorem 9 Let $a(1,1)=a(2,1)=a(1)$ and $a(1,2) \neq a(2,2)$.

(i) If

$$
F^{j}\left(\bar{X}^{1}, \bar{X}^{2}\right) \geq \bar{\alpha}^{j} \text { for } j=1,2,
$$

where

$$
F^{j}\left(\xi^{1}, \xi^{2}\right)=\alpha_{a(1)}^{j}\left(\frac{1}{N_{a(1)}^{0}+\xi^{\hat{j}}}-\frac{\xi^{\hat{j}}}{\left(N_{a(1)}^{0}+\xi^{j}\right)^{2}}\right), \bar{\alpha}^{j}=\frac{\alpha_{a(j, 2)}^{j}}{N_{a(j, 2)}^{0}}
$$

then $\left(\mathcal{T}_{a(1)}^{1}, \mathcal{T}_{a(1)}^{2}\right)$ is optimal.

(ii) If

$$
F^{1}\left(\bar{X}^{1}, \bar{X}^{2}\right)<\bar{\alpha}^{1} \text { and } F^{2}\left(\bar{X}^{1}, \bar{X}^{2}\right)>\bar{\alpha}^{2}
$$

then $\left(T^{1}, T^{2}\right)$ is optimal, where $T^{2}=\mathcal{T}_{a(1)}^{2}, T^{1}=\left(t, \bar{T}^{1}-t, 0, \ldots, 0\right), t=$ $\max \{0, T\}$ and $T$ is the maximal root of the equation:

$$
\alpha_{a(1)}^{1}\left(\frac{1}{N_{a(1)}^{0}+\alpha_{a(1)}^{1} \bar{T}^{2}}-\frac{\alpha_{a(1)}^{1} \bar{T}^{2}}{\left(N_{a(1)}^{0}+\alpha_{a(1)}^{2} T\right)^{2}}\right)=\bar{\alpha}^{1} .
$$

(iii) The case $F^{1}\left(\bar{X}^{1}, \bar{X}^{2}\right)>\bar{\alpha}^{1}$ and $F^{2}\left(\bar{X}^{1}, \bar{X}^{2}\right)<\bar{\alpha}^{2}$ is symmetric to (ii).

(iv) If

$$
F^{j}\left(\bar{X}^{1}, \bar{X}^{2}\right)<\bar{\alpha}^{j} \text { for } j=1,2
$$

then $\left(T^{1}, T^{2}\right)$ is optimal, where $T^{j}=\left(x^{j} / \alpha_{a(1)}^{j}, \bar{T}^{j}-x^{j} / \alpha_{a(1)}^{j}, 0, \ldots, 0\right)$ and $\left(x^{1}, x^{2}\right)$ is the positive solution of the system $F^{j}\left(x^{1}, x^{2}\right)=\bar{\alpha}^{j}, j=1,2$.

This theorem allows us to depict (see Figure 2) in coordinate system $\left(\bar{X}^{1}, \bar{X}^{2}\right)$ how $\mathrm{NE}$ and cooperative equilibria differ depending on the power signal users can apply. The area $\left\{\left(\bar{X}^{1}, \bar{X}^{2}\right): X^{j} \leq A^{j}, j=1,2\right)$, where the pure equilibrium $\left(\mathcal{T}_{a(1)}^{1}, \mathcal{T}_{a(1)}^{2}\right)$ is NE, is essentially larger than the area $\left\{\left(\bar{X}^{1}, \bar{X}^{2}\right): F^{j}\left(\bar{X}^{1}, \bar{X}^{2}\right) \geq\right.$ $\left.\bar{\alpha}^{j}\right\}$ where it is also the cooperative equilibrium. Meanwhile the area $\left\{\left(\bar{X}^{1}, \bar{X}^{2}\right)\right.$ : $\left.F^{j}\left(\bar{X}^{1}, \bar{X}^{2}\right)>\bar{\alpha}^{j}\right\}$ where in cooperative behavior the users prefer to employ mixed strategies include the corresponding area for $\mathrm{NE}$ as well as a part of the area where in NE a user is squeezed from the best sub-carrier. Thus, in the cooperative scenario in comparison with the non-cooperative scenario the area where users prefer to employ pure strategies is essentially narrower. 
The conditions that $\left.F^{j}\left(\bar{X}^{1}, \bar{X}^{2}\right)>\bar{\alpha}^{j}\right\}$ and $\bar{X}^{j}<\bar{A}^{j}$ can be interpreted as if user $j$ has a small signal to transmit for cooperative and selfish scenarious respectively meanwhile the conditions that $\left.F^{j}\left(\bar{X}^{1}, \bar{X}^{2}\right)<\bar{\alpha}^{j}\right\}$ and $\bar{X}^{j}>\bar{A}^{j}$ can be interpreted as if user $j$ has a big signal to transmit. If one of the users has a big signal to transmit (called a stronger user) for both scenarious meanwhile his rival has small signal to transmit (weaker user) then behaviour of the stronger user drastically changes in cooperative plot compare to selfish one. Namely, in selfish one the stronger user squeezes the weaker one from the best channel meanwhile in cooperative he allows the weaker user employs the best channel and himself prefers to apply a mixed strategy. Finally note that the lines on Figure 2 can be also interpreted as switching lines of cost of anarchy.

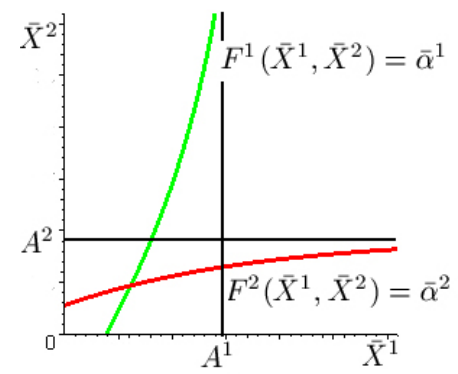

Fig. 2. Cooperative and Nash equilibria

\section{Appendix}

Proof of Theorem 6. Since $a(1,2) \neq a(1,2)$ the users do not have colluded interests in the second by quality sub-carriers which are different for them. Also, $T_{a(1)}^{1}>0$ and $T_{a(1)}^{2}>0$ since the users apply mixed strategies. Thus, by Theorem $1, \omega^{1}=\alpha_{a(1,2)}^{1} / N_{a(1,2)}^{0}, \omega^{2}=\alpha_{a(2,2)}^{2} / N_{a(2,2)}^{0}$, and

$$
\frac{\alpha_{a(1)}^{1}}{N_{a(1)}^{0}+\alpha_{a(1)}^{2} T_{a(1)}^{2}}=\omega^{1}, \quad \frac{\alpha_{a(1)}^{2}}{N_{a(1)}^{0}+\alpha_{a(1)}^{1} T_{a(1)}^{1}}=\omega^{2} .
$$

Solving these equations for $T^{1}$ and $T^{2}$ implies (3).

Proof of Theorem 9. Since $a(1,2) \neq a(2,2)$ and $a(1,1)=a(2,1)$ the optimal strategy $T^{j}, j=1,2$ has to be of the form

$$
T_{i}^{j}= \begin{cases}t^{j} & \text { for } i=a(1) \\ \bar{T}^{j}-t^{j} & \text { for } i=a(j, 2) \\ 0 & \text { otherwise }\end{cases}
$$


where $t^{j} \in\left[0, \bar{T}^{j}\right]$ and the optimal $t^{1}$ and $t^{2}$ are the ones which maximize the following function

$$
v\left(t^{1}, t^{2}\right)=\frac{\alpha_{a(1)}^{1} t^{1}}{N_{a(1)}^{0}+\alpha_{a(1)}^{2} t^{2}}+\frac{\alpha_{a(1)}^{2} t^{2}}{N_{a(1)}^{0}+\alpha_{a(1)}^{1} t^{1}}+\sum_{i=1}^{2} \frac{\alpha_{a(i, 2)}^{i}}{N_{a(i, 2)}^{0}}\left(\bar{T}^{i}-t^{i}\right) .
$$

To find the optimal $t^{1}$ and $t^{2}$, we need to calculate the derivatives of $v$ with respect to $t^{1}$ and $t^{2}$ :

$$
v_{j}\left(t^{1}, t^{2}\right):=\frac{\partial v}{\partial t^{j}}=\frac{\alpha_{a(1)}^{j}}{N_{a(1)}^{0}+\alpha_{a(1)}^{t^{\hat{j}}} t^{\hat{j}}}-\frac{\alpha_{a(1)}^{1} \alpha_{a(1)}^{2} t^{\hat{j}}}{\left(N_{a(1)}^{0}+\alpha_{a(1)}^{t^{j}} t^{\hat{j}}\right)^{2}}-\frac{\alpha_{a(j, 2)}^{j}}{N_{a(j, 2)}^{0}}, j=1,2 .
$$

It is clear that

$$
v_{j}\left(t^{1}, t^{2}\right)=\tilde{v}_{j}\left(\xi^{1}, \xi^{2}\right):=F^{j}\left(\xi^{1}, \xi^{2}\right)-\bar{\alpha}^{j},
$$

where $\xi^{j}=\alpha_{a(1)}^{j} t^{j}$ and $\xi^{j} \in\left[0, \tilde{T}^{j}\right]$ with $\tilde{T}^{j}=\alpha_{a(1)}^{j} \bar{T}^{j}, j=1,2$.

$\tilde{v}_{j}$ has the following properties:

$$
\begin{gathered}
\tilde{v}_{j} \text { is increasing on } \xi^{j} \text { and decreasing on } \xi^{\hat{j}}, \\
\tilde{v}_{1}(t, 0)=\frac{\alpha_{a(1)}^{1}}{N_{a(1)}^{0}}-\frac{\alpha_{a(1,2)}^{1}}{N_{a(1,2)}^{0}}>0, \tilde{v}_{2}(0, t)=\frac{\alpha_{a(1)}^{2}}{N_{a(1)}^{0}}-\frac{\alpha_{a(2,2)}^{2}}{N_{a(2,2)}^{0}}>0 \text { for } t \geq 0 .
\end{gathered}
$$

By (10) the points $(0,0),(0, \xi),(\xi, 0)$ cannot be the maximal ones.

In Figure 3 we depict signs of $\tilde{v}_{1}$ and $\tilde{v}_{2}$ in the coordinate system $\left(\xi^{1}, \xi^{2}\right)$. Namely, the region $(+,+)$ presents the set $\left\{\left(\xi^{1}, \xi^{2}\right): \tilde{v}_{1}\left(\xi^{1}, \xi^{2}\right)>0, \tilde{v}_{2}\left(\xi^{1}, \xi^{2}\right)>\right.$ $0\}$, the region $(-,-)$ is $\left\{\left(\xi^{1}, \xi^{2}\right): \tilde{v}_{1}\left(\xi^{1}, \xi^{2}\right)<0, \tilde{v}_{2}\left(\xi^{1}, \xi^{2}\right)<0\right\}$ and so on. Then, if $\left(\tilde{T}^{1}, \tilde{T}^{2}\right) \in(-,-)$ which corresponds to $(5)$ then the maximum is at $\left(\tilde{T}^{1}, \tilde{T}^{2}\right)$ and (i) is proved. If $\left(\tilde{T}^{1}, \tilde{T}^{2}\right) \in(+,+)$ which corresponds to (8) then the maximum is at $\left(\xi^{1}, \xi^{2}\right)$ which is the unique positive solution of the system $\tilde{v}_{i}\left(\xi^{1}, \xi^{2}\right)=0, i=$ 1,2 and (iv) is proved. Similarly, the cases $\left(\tilde{T}^{1}, \tilde{T}^{2}\right) \in(+,-)$ and $\left(\tilde{T}^{1}, \tilde{T}^{2}\right) \in$ $(-,+)$ correspond to $(8)$ respectively and are investigated similarly.

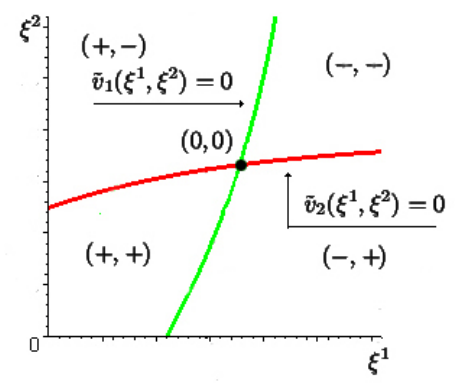

Fig. 3. Signs of $\tilde{v}_{1}$ and $\tilde{v}_{2}$ 


\section{References}

1. S. Adlakha, R. Johari, and A. Goldsmith, "Competition in Wireless Systems via Bayesian Interference Games", in Arxiv.org, no.0709.0516.

2. E. Altman, K. Avrachenkov, and A. Garnaev, "Closed form solutions for symmetric water filling games", in Proc. of IEEE INFOCOM 2008.

3. E. Altman, K. Avrachenkov, and A. Garnaev, "Fair resource allocation in wireless networks in the presence of a jammer", in Proc. of ValueTools 2008, 2008.

4. R. Etkin, A. Parekh and D. Tse, "Spectrum Sharing in Unlicensed Bands", IEEE J. on Selec. Areas Comm., v.25, no.3, pp.517-528, April 2007.

5. H. Holma and A. Toskala. WCDMA for UMTS.

6. H. Ji and C.-Y. Huang, "Non-cooperative uplink power control in cellular radio systems", Wireless Networks, v.4, pp.233-240, 1998.

7. S. L. Kim, Z. Rosberg, and J. Zander. Combined power control and transmission selection in cellular networks. In Proceedings of IEEE Vehicular Technology Conference, Fall 1999.

8. I. Koo, J. Ahn, H. A. Lee, and K. Kim. Analysis of Erlang capacity for the multimedia DS-CDMA systems. IEICE Trans. Fundamentals, E82-A(5):849-855, May 1999.

9. D. Tse and P. Viswanath, Fundamentals of Wireless Communication, Cambridge University Press, 2005. 